\title{
PRÁCTICAS CAMPESINAS EN EMPLAZAMIENTOS AGRÍCOLAS FORMATIVOS. EL CASO DEL ALTO JUAN PABLO (BELÉN, CATAMARCA)
}

\section{PEASANT PRACTICES IN RURAL LOCATIONS OF THE FORMATIVE PERIOD: THE CASE OF ALTO JUAN PABLO (BELÉN, CATAMARCA).}

\author{
Mariana Maloberti \\ E Instituto de Arqueología y Museo - Instituto Superior de Estudios Sociales (CONICET - UNT). \\ E-mail: marianamaloberti@yahoo.com.ar
}

Presentado el: 26/07/2013 - Aceptado 23/04/2014

\begin{abstract}
Resumen
A través de la investigación que aquí presentamos, buscamos indagar en las prácticas campesinas que configuraron, durante el Formativo, un emplazamiento exclusivamente agrícola ubicado en el sector septentrional del valle El Bolsón: Alto Juan Pablo. El estudio de estas prácticas fueron abordadas desde múltiples aproximaciones, entre las cuales incluimos, en primer lugar el análisis de la configuración de las estructuras agrícolas y la consiguiente generación de secuencias constructivas de los espacios productivos. En segundo lugar, aplicamos algunas técnicas para conocer las actividades de los campesinos que habitaron el Alto Juan Pablo, lo cual nos ayudó a aproximarnos a su vida cotidiana en torno a la producción de alimentos. Entre estas técnicas contamos con el estudio del conjunto lítico recuperado en superficie y el análisis múltiple de microfósiles provenientes de sedimentos de estructuras agrícolas y su caracterización pedológica. A través de estos abordajes, logramos tener una imagen de las prácticas por medio de las cuales el sitio fue corporizado, así como también de la forma particular de habitar ese espacio.
\end{abstract}

Palabras claves: Prácticas campesinas, Valle de El Bolsón, Paisajes agrarios

\footnotetext{
Abstract

In this article, we present a study focusing on productive practices that shaped an exclusively agricultural site, located at the north of El Bolsón valley: Alto Juan Pablo. This study was carried out from multiple approaches including, in first place, the analysis of the configuration of agricultural structures and the consequent generation of construction sequences of these productive spaces. Second, we include some technical strategies for understanding the activities of the peasants who
} 
inhabited the Alto Juan Pablo, and their everyday life around food production. These strategies include the study of lithic assemblage and a multiple analysis of microfossils from sediments recovered from the agricultural structures and their pedological characterization. Through these research, we intend to think the practices through which the site was embodied and the particular way of dwell that space.

Key words: Peasants practices, El Bolsón valley, Agrarian landscape

\section{Introducción}

Como fue observado por algunos autores, en los estudios vinculados a la agricultura prehispánica del Noroeste Argentino (NOA en adelante), no fueron numerosos los casos que centraron sus análisis en los sitios de producción mismos, sin embargo aquellos que de hecho incorporaron estos espacios a la investigación, conllevaron un avance claro en lo que a la agricultura prehispánica refiere (por ejemplo Albeck 1993, Álvarez 2001, Korstanje 2005, Quesada 2007, Caria et al. 2007, Figueroa 2008, Díaz 2009, Franco Salvi 2012, entre otros). Este posicionamiento de los ámbitos de producción en el centro de la escena de la investigación, frecuentemente fue acompañado por la incorporación de nuevas estrategias metodológicas y teóricas que permitieron, en algunos casos, rever algunos de los supuestos tradicionales asociados a la historia agraria del NOA (Korstanje et al. 2012). Un ejemplo de esto lo constituyó el caso del valle de El Bolsón (Departamento Belén, Catamarca), donde las investigaciones centradas en la producción campesina durante el Formativo, llevaron a Korstanje a incorporar el análisis múltiples de microfósiles provenientes de sedimentos de estructuras productivas, como una de las vías para su estudio (Korstanje 2002, 2005, 2009). Estos trabajos contribuyeron a la comprensión de la producción campesina durante el Primer Milenio en los valles altos catamarqueños, un área sub - representada en la literatura arqueológica, sobre todo en relación a la Puna y valles bajos (Korstanje 2010).

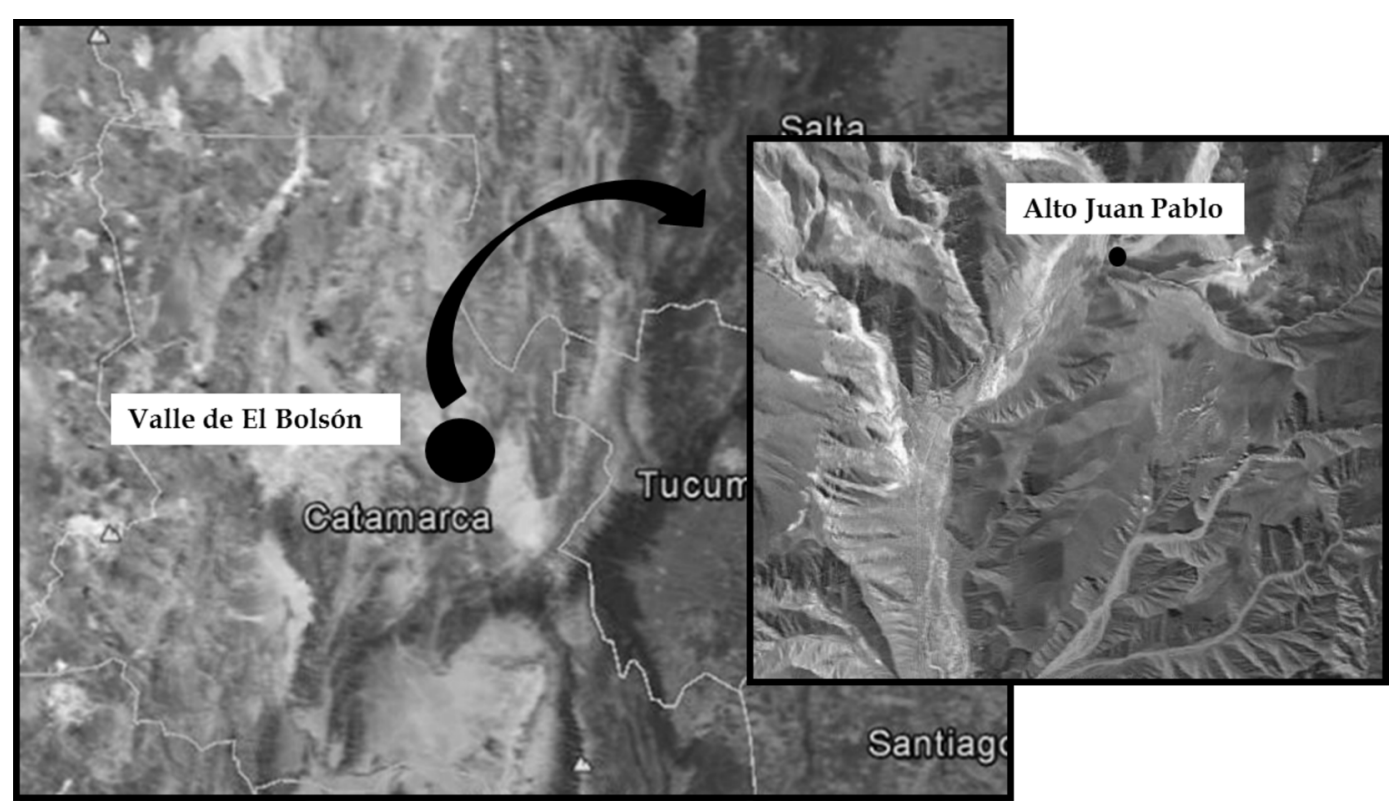

Figura 1. Ubicación del valle de El Bolsón (izquierda) y del sitio Alto Juan Pablo (derecha) en imagen tomada de Google Earth 
En esta misma línea, en el trabajo que aquí presentamos buscamos indagar en las prácticas campesinas que configuraron, durante el Formativo, un emplazamiento exclusivamente agrícola ubicado en el sector septentrional del valle El Bolsón: Alto Juan Pablo (Figura 1). Para ello, recurrimos a diferentes vías analíticas, entre las cuales incluimos el análisis de microfósiles provenientes de sedimentos muestreados en canchones de cultivo, tomando como base interpretativa y metodológica las propuestas que Korstanje y Cuenya vienen desarrollando desde el año 2006 (Korstanje y Cuenya 2008, 2010, Korstanje et al. 2013). Estos estudios, complementados con el análisis de las técnicas constructivas de las estructuras y su secuencia de crecimiento, como así también el estudio técnico - morfológico del conjunto lítico, nos permitieron reconocer las formas particulares de habitar ese espacio.

\section{Algunas consideraciones teóricas}

Para abordar este trabajo, partimos de las concepciones vinculadas al estudio del "paisaje", tal como fueron desarrolladas por Ingold $(1993,2000)$. La concepción de "paisaje" según el autor mencionado propone no circunscribirse a lo que llamó "visiones naturalistas" en las que se presupone al paisaje como algo natural o marco externo de las actividades humanas; como tampoco a las "visiones culturalistas" en las que el paisaje aparece como construcción cognitiva del espacio, es decir como una imagen en el imaginario; sino más bien propone desmarcarse de estos opuestos. Para ello, define el paisaje como el registro duradero o testimonio de la vida de pasadas generaciones que lo han habitado y dejaron allí algo de sí mismo, es decir como el registro de la corporización de los sujetos que allí han vivido (Ingold 1993). De esta manera, es entendido como un espacio vivido, habitado, espacio que se vuelve parte de nosotros, tanto como nosotros parte de él. Como parte de su propuesta, Ingold invita a su estudio a través de indagar en su temporalidad, la cual emerge del proceso mismo de habitar un paisaje y concretamente a través de las actividades de aquellos que lo habitan, refiriéndose a este conjunto de actos como "Taskscapes" (Ingold 1993). Creemos que este último concepto, que refiere a los "actos del habitar", nos permite concebir a las prácticas cotidianas como una vía a través de la cual pensar cómo fue configurándose el sitio Alto Juan Pablo durante el Primer Milenio.

Por otro lado, pensamos que la incorporación de esta perspectiva conlleva una comprensión más acabada de los procesos históricos, alejándonos de perspectivas esencialistas que conciben a este proceso como heredado inminentemente desde la cultura o pautado de manera determinante por el ambiente, sin con esto pretender minimizar las influencias ejercidas por ambos.

Entonces, buscamos comprender las formas particulares de habitar un emplazamiento agrícola, a través de las prácticas campesinas que allí tuvieron lugar durante el Formativo. Si proponemos un estudio arqueológico de estas prácticas es que estamos asumiendo que pueden ser abordadas desde la materialidad arqueológica. Así, consideramos que las prácticas sociales pueden ser interpretativamente reconstruidas desde la arqueología, en virtud de que tenemos acceso a su materialidad. Como lo expresan Shanks y Tilley (1992), la materia inerte es transformada por las prácticas sociales en un bien cultural (desde una herramienta a una obra de arte). Esta transformación se constituye en la objetivación de la práctica social, práctica que a su vez está impresa en esa cultura material. Una vez objetivada la práctica en una forma material, esta se inserta en el contexto de prácticas futuras a las cuales influye, de aquí se deriva la idea de la cultura material como estructurada y al mismo 
tiempo estructurante de las prácticas. En este planteo podemos trazar claros paralelos entre la idea de Shanks y Tilley de la cultura material como objetivación de las prácticas con la de Ingold del paisaje como un taskscape en su forma corporizada.

Como ya adelantamos, el estudio de estas prácticas fueron abordadas desde múltiples aproximaciones, entre las cuales incluimos, en primer lugar al análisis de la configuración de las estructuras agrícolas y la consiguiente generación de secuencias constructivas de los espacios productivos (Quesada y Maloberti 2012). En segundo lugar, aplicamos algunas técnicas para conocer las actividades de los campesinos que habitaron el Alto Juan Pablo, lo cual nos ayudó a aproximarnos a su vida cotidiana en torno a la producción de alimentos. Entre éstas estrategias contamos con el estudio del conjunto lítico recuperado en superficie y el análisis múltiple de microfósiles provenientes de sedimentos de estructuras agrícolas y su caracterización pedológica.

Finalmente, si bien en este trabajo abordamos el estudio de un caso particular, consideramos que el mismo puede contribuir a pensar los paisajes agrarios formativos del sector septentrional del valle El Bolsón.

\section{El entorno ambiental del sitio}

De acuerdo a lo expresado anteriormente, no consideramos al entorno ambiental como escenario pasivo en el que se llevan a cabo las actividades humanas, pero tampoco como producto de construcciones cognitivas de las sociedades (Descola 2001). Coincidimos con autores como Haber (1999) cuando afirman que pretender que los procesos históricos en nada se vinculan con las condiciones naturales sería inútil. De esta manera, el ambiente lejos de estar naturalmente separado de las sociedades, es materia de apropiación y modificación de las mismas a través de vínculos dialógicos entablados entre las estructuras ambientales y las prácticas sociales. Es por esto que consideramos importante presentar una breve caracterización de uno de los términos que participan en ese diálogo: la estructura ambiental.

\section{Caracterizando la estructura ambiental}

Korstanje (2005) presenta al valle de El Bolsón como un valle alto en relación al área valliserrana (2900 a 2500 m.s.n.m), angosto (ancho máximos de $2 \mathrm{Km}$ ) y extensión total de $21 \mathrm{Km}$ corriendo de norte a sur. Según Aschero y Korstanje (1996) se trata de una zona transicional entre la región valliserrana y la Puna.

El sector septentrional del valle corresponde a lo que Burkart et al. (1999) definieron como eco - región de Monte de Sierras y Bolsones y una angosta franja a la eco - región de Puna. De acuerdo a los estudios paleoambientales conducidos por Ortiz (2001) y Madozzo (2009), a partir del análisis de micromamíferos hallados en las cuevas de Los Viscos y Las Máscaras respectivamente (ubicadas en el sector central del valle), proyectan para El Bolsón en general una reconstrucción climática según la cual en los últimos dos milenios, el clima habría oscilado dentro de los parámetros actuales, con excepción de un registro de incremento de humedad entre el 1000 y el 500 AP, que se corresponde con el Óptimo Climático Mundial. Otra excepción mencionada por los autores, se ubica entre los años 550 y $250 \mathrm{AP}$, indicando una mayor aridez, lo cual coincide con la Pequeña Edad de Hielo (Ortiz 2001, Madozzo 2009). 
Específicamente hablando del sitio Alto Juan Pablo, el mismo está ubicado en una pequeña planicie sobre una elevación pequeña, aislada y de fuerte pendiente (valor promedio de pendiente oeste - este $=3^{\circ}=6.6 \%$; valor promedio de pendiente sur - norte $=3.83^{\circ}$ $=8.51 \%)$. Esta elevación está constituida por materiales terciarios de roca metamórfica cubiertos por derrubios cuaternarios. En lo respectivo a los límites de dicha elevación, al norte limita con un sector de medanales, al igual que al este; mientras que al oeste colinda con la Ruta Provincial No 43 y hacia el sur, se halla el rió El Bolsón junto al actual poblado de Los Nacimientos de San Antonio. La mesada en la que se encuentran las estructuras del sitio, habrían constituido una antigua terraza relictual del Río El Bolsón. Parte de la misma se halla cubierta por un médano, cuya lengua de arena se extiende en dirección oeste, cubriendo parte del sitio, lo cual dificultó nuestras tareas de prospección en el lugar. Según observaciones realizadas por Kulemeyer et al. (2012), se trataría de depósitos eólicos que constituyen un frente de dunas que avanza desde la Puna con rumbo WNW - ESE. Este avance se habría producido con posterioridad a la construcción del sitio, ya que cubre parte del mismo (Kulemeyer et al. 2012)

Finalmente, para evaluar la erosión in situ, se decidió aplicar el índice de Morgan (1986), no sólo por su sencillez, sino también por su especial énfasis en la evaluación de erosión del suelo. En nuestro caso, circunscribiéndonos al sitio, encontramos una erosión tipo 3 (severa) a 4 (grave). Si tomamos un área mayor (o sea toda la elevación) la situación erosiva se agrava a un tipo 5 (muy grave), siendo los pedestales de erosión de más de $60 \mathrm{~cm}$, los surcos, las marcas de crioclastía en rocas y cuevas de roedores y reptiles, algunos de los rasgos que atestiguan los procesos erosivos en el lugar.

\section{Configuración arquitectónica}

El Alto Juan Pablo está conformado por una sucesión de seis estructuras agrícolas de piedra (E3 - E4 - E5 - E6 - E7 - E8), algunas de ellas sub-circulares y otras de forma más cuadrangular (Figura 2). Estos recintos se hallan conectados entre sí, sin solución de continuidad, extendiéndose longitudinalmente en forma escalonada siguiendo la pendiente general de este a oeste. Sin continuidad constructiva con las anteriores pudimos detectar una séptima estructura (E10) que evidenciaba características distintivas a las recién mencionadas, como ser su forma claramente circular, mejor conservación (de hecho sus muros de más de un metro de alto continúan en pie, con derrumbes poco significativos), entre otras. A través de entrevistas realizadas a los habitantes de la localidad de Los Nacimientos de San Antonio, se nos informó acerca del carácter actual de su construcción, llevada a cabo hace 50 años, con la finalidad de ser utilizada como corral, actualmente en desuso, razón por la cual la excluimos de nuestro análisis.

Para registrar la configuración arquitectónica del sitio, llevamos a cabo un relevamiento planialtimétrico, acompañado por una prospección superficial pedestre. En ambas instancias buscamos, en primer lugar realizar un relevamiento de detalle de las características constructivas de los canchones como así también la detección de posibles canales de riego ya que nos encontramos con un diseño de las estructuras articuladas, muy común de ser asociado a una estrategia para distribuir el agua de un canchón a otro, sin embargo no se hallaba a simple vista ninguna estructura que podía asociarse con la práctica de riego (por ejemplo canales). Entendimos que, para poder comenzar a discutir la presencia de un diseño de estructuras agrícolas asociada al riego en un lugar donde éste no se hace evidente, primero debíamos cerciorarnos de que no había estructuras vinculadas al manejo del agua. Es así 
como decidimos en nuestras prospecciones (sobre todo en la conducida en el área exterior a las estructuras), enfocarnos en la búsqueda de dichos rasgos. Por otro lado, pensamos que si realmente el área agrícola estaba siendo regada, podríamos esperar encontrar la toma en el curso de agua cercano al sitio, razón por la cual condujimos un recorrido pedestre acompañando al río. Ninguna de las prospecciones realizadas arrojó resultados positivos que permitan evidenciar el riego.

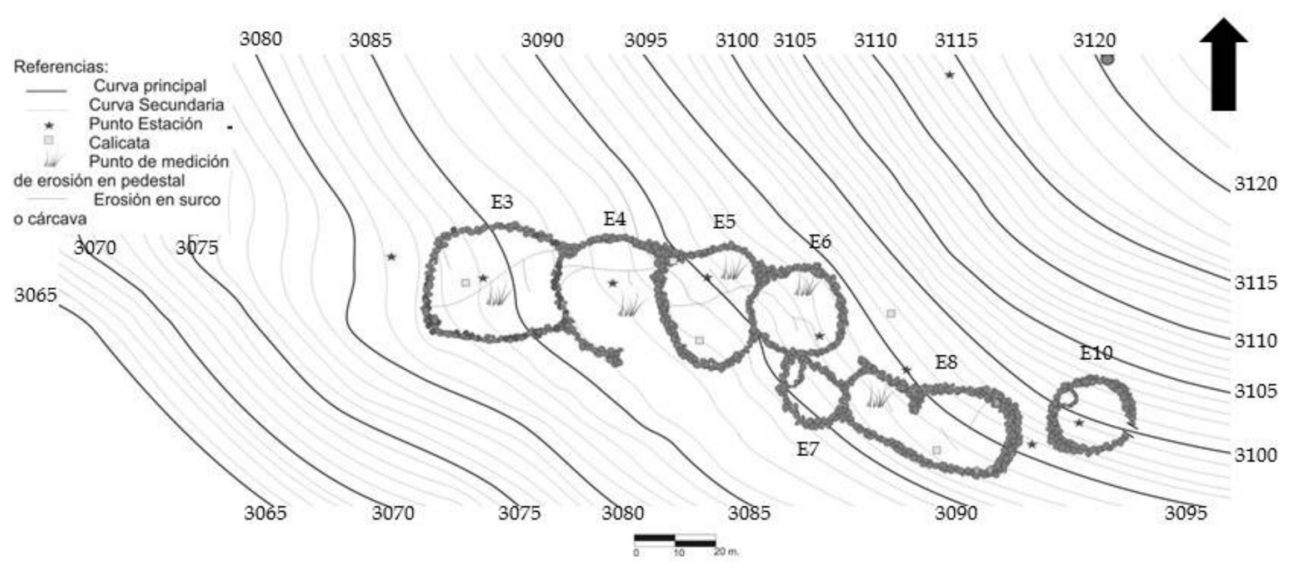

Figura 2. Cartografía del sitio

Para la descripción de las estructuras, se registró: forma y tamaño, la elevación máxima y mínima y ancho de los muros, presencia de derrumbes y su localización, presencia de elementos intersticiales en las roca, ordenamiento de rocas en un área muestra de $1 \mathrm{~m} 2$ (tamaño, forma y cantidad de rocas apiladas), etc. En general observamos en todos los casos, muros sólidos y anchos (entre 0,80 a 1,10 m), construidos con diferentes tamaños de clastos no dispuestos ordenadamente (es decir no depositados según su rango de tamaño) y que pueden encontrarse en las inmediaciones del sitio, habiéndose seleccionado tanto clastos angulosos como redondeadas para la construcción.

A partir de la homogeneidad en los rasgos descriptos en las diferentes estructuras, pudimos observar una continuidad en sus características constructivas. Así, no hay ningún elemento que nos indique una modificación en las decisiones de selección de las rocas para construir los muros o de construcción de los mismos.

Por otro lado, otro de los objetivos del relevamiento y descripción en detalle de los canchones consistió en realizar un ordenamiento en secuencia de los mismos, como una manera de hipotetizar la forma en que ese paisaje agrario habría crecido. La disposición yuxtapuesta de las estructuras hacía posible sugerir una secuencia constructiva en términos relativos, la cual implicaba que algunos de los canchones habrían sido construidos antes que otros, pudiendo ser el tiempo transcurrido entre la construcción de uno de ellos y el subsiguiente, de días, años o siglos, razón por la cual lo que pretendemos reconstruir son las relaciones estratigráficas de adosamiento ordenadas en una secuencia de crecimiento (Quesada y Maloberti 2012). Para esto se prestó especial atención a la forma en que se adosa una estructura a la otra a través del muro común. 
En otras palabras, dadas dos estructuras adosadas, se busca determinar a cuál de ellas perteneció, en primera instancia el muro compartido, y cuál se yuxtapone a la anterior aprovechándolo oportunamente.

De esta manera se define como primera estructura a E6, la cual se trata de un canchón comparativamente más pequeño a los restantes, cuya forma casi circular está mejor definida y se ubica en el centro de la serie de estructuras. Así, nuestra secuencia habría nacido desde un punto central extendiéndose constructivamente en dos direcciones (este y oeste). Continuando con la secuencia (ya no concebida como unidireccional), ésta pudo continuar con la incorporación de E5 (hacia el este) y/o E7 (hacia el oeste), por lo cual ambas constituirían una posible segunda fase estratigráfica de adosamiento, subsiguiente a la construcción de E6.

La tercera fase estratigráfica podría estar definida por el adosamiento de E4 (hacia el este) y/o E8 (oeste). De esta manera concluye en la tercera fase estratigráfica de la secuencia, las construcciones que se extienden en dirección oeste, por lo que la cuarta fase está únicamente representada por E3 (este), último canchón con la que finaliza la secuenciación relativa de las estructuras articuladas.

Analizando la secuencia final recién propuesta (Figura 3), el sitio aparece configurado a través de un proceso de construcción en que la expansión del sector agrícola se orienta por el aprovechamiento de espacios dejados por las estructuras primarias, resultando de estas decisiones constructivas, un modelo irregular.
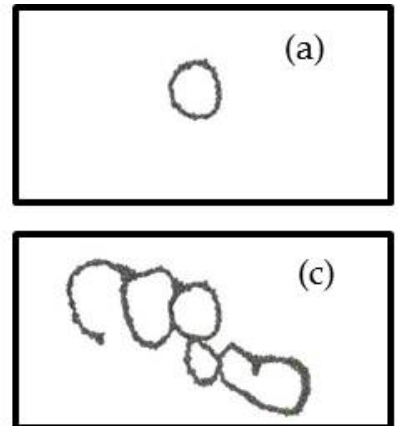

(c)

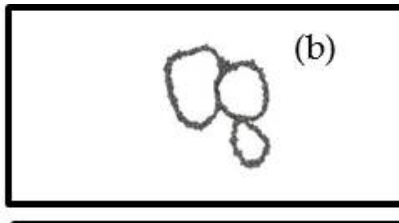

(d)

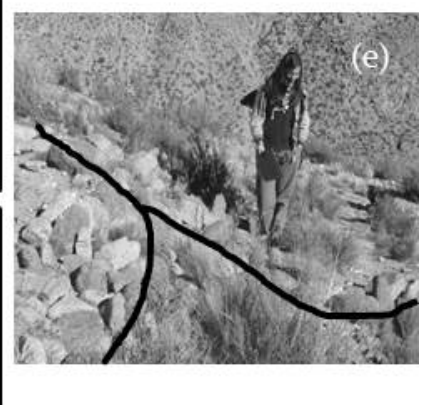

Figura 3. Secuencia de crecimiento del sector construido del sitio. (a) Primera fase estratigráfica: E6; (b) Segunda fase estratigráfica: E5 _E6_E7; (c) Tercera fase estratigráfica: E4_E5_E6_E7_E8//;

(d) Cuarta fase estratigráfica: //E3_E4_E5_E6_E7_E8//; (e) Detalle de adosamiento de un sector del muro de E6 con un sector del muro de E5

\section{El Conjunto lítico y algunas consideraciones acerca de la cronología}

Con el objeto de adscribir temporalmente el sitio, se condujo una prospección sistemática con muestreo estratificado en la cual incluimos no solo el sitio sino también los sectores aledaños, y en la cual se recolectó todo el material considerado cronológicamente diagnóstico. Como es esperable para los sectores productivos, el conjunto cerámico recuperado no es muy numeroso (menos de 50 fragmentos) ${ }^{1}$, a pesar de lo cual, a partir de la presencia de cerámicas con características afines a las definidas para momentos Formativos en el valle (por ejemplo Río Diablo [Korstanje 2005]), es que propusimos una ubicación temporal del sitio dentro del Primer Milenio. Esta idea se reforzó por la completa ausencia de materiales 
adscribibles al Período de Desarrollo Regional y por las observaciones de características del conjunto lítico recuperado, no discordantes con momentos Formativos (tipo de puntas de proyectil, el tipo de lascado marginal de los artefactos, preponderancia de artefactos unifaciales y compuestos, etc.) (Mauri, com. personal).

Sin embargo el conjunto lítico de superficie recuperado no fue solo estudiado como una aproximación más para ubicar temporalmente el sitio, sino que también incorporamos una caracterización del mismo con el objeto de reconocer qué actividades podrían haberlo originado y así aportar un elemento más para reconstruir el Taskscape de este emplazamiento agrícola. Para ello, junto a Eduardo Mauri, seguimos los criterios técnico - morfológicos propuestos por Aschero $(1975,1983)$ y Aschero y Hocsman $(2004)$, determinando las materias primas macroscópicamente.

\section{Resultados del Análisis}

El conjunto lítico está conformado por un total de 21 artefactos y 48 desechos de talla (Número Mínimo de Desechos: 30, representando un 62,5 \% del total de los desechos). Entre las clases artefactuales identificadas, encontramos: artefactos compuestos (n: 4), punta entre muescas (n: 3), raspadores (n: 2), raederas (n: 2), Chopping Tool (n: 2), raclette (n: 1), cuchillo (n: 1), denticulado (n: 1) y puntas de proyectil (n: 5).

El tamaño de las piezas es variable encontrándose artefactos con rangos de tamaño "muy grande" pero otros "pequeños". Por otro lado registramos una uniformidad respecto a la relación ancho/largo, ya que los módulos "anchos" predominaron sobre los "largos".

Con respecto al conjunto de desechos de talla, éste se presenta como altamente homogéneo ya que el 100\% corresponde a lascas internas y angulares. Sin embargo los tamaños varían de microlascas a lascas grandes aunque sus anchos son relativamente uniformes predominando las lascas anchas a muy anchas. Por otro lado en 17 desechos de talla observamos posibles indicios de reactivación de filos.

En lo que a la materia prima refiere, para determinar el carácter local o no local de la misma, hemos tomado los trabajos de Somonte (2004) y Babot y Larrahona (2001), quienes llevaron a cabo estudios de fuentes de aprovisionamiento en el sector septentrional del valle El Bolsón. De esta manera, el conjunto lítico del Alto Juan Pablo presenta un alto predominio de materias locales, tanto para artefactos como para los desechos. Para, estos últimos, el material predominante es el basalto ${ }^{2}$, siendo el único material no local representado la obsidiana. En los artefactos, la materia prima preponderante corresponde a la andesita y con valores muy cercanos le sigue el basalto (sobre este último se confeccionó la totalidad de puntas que conforman el conjunto lítico excluyendo un ápice de obsidiana), siendo también la obsidiana el único recurso no local representado. A diferencia de los artefactos de basalto, los confeccionados en andesita aparecen descartados por fractura o embotamiento sin reavivación de los filos, es decir sin una inversión de trabajo para su reactivación.

Por otro lado, observamos que en la confección de artefactos de mayor tamaño hubo una clara preferencia por el uso de recursos locales y de alta disponibilidad en las inmediaciones al sitio. Así, los instrumentos más grandes registrados en el conjunto fueron confeccionados sobre metamórficas (por ejemplo el chopping tool). 
Finalmente el hecho de que el conjunto de desechos de talla se halla claramente representado por lascas internas, nos hace pensar que no se llevaron a cabo en el sitio actividades de reducción primaria sino más bien tareas de regularización, mantenimiento o reciclaje de artefactos. Es decir más que una formatización por talla en el sitio, podemos pensar en un reacondicionamiento de instrumentos confeccionados fuera de él. Por otra parte, la clara preponderancia de lascas anchas puede sugerir que se estaban reactivando artefactos grandes. Con respecto a esto último, podemos aventurarnos a pensar que algunos de esos artefactos de gran tamaño que se podrían estar reactivando pueden corresponder a aquellos utilizados durante la labranza, raleo, surcado, etc. (los cuales presentan módulos de tamaño considerables [ver por ejemplo Escola et al. 2012]).

\section{Análisis de microfósiles en sedimentos}

Las muestras de sedimento, a partir de las cuales realizamos nuestros análisis, fueron tomadas siguiendo el protocolo mencionado por Pearsall (2000) y Piperno (1988), muestreándose tres de los seis canchones del sitio (Figura 4), a las cuales sumamos una cuarta zona de muestreo correspondiente al sector extra - sitio ${ }^{3}$, el cual es tomado como referente de las condiciones naturales del suelo (o por lo menos no afectado por las actividades agrícolas pasadas). Las muestras colectadas (siguiendo niveles artificiales cada $15 \mathrm{~cm}$ ) fueron luego procesadas y analizadas para determinar las características físicas de los sedimentos y para el estudio múltiple de microfósiles. Para lo primero, se llevaron a cabo dos tipos de análisis ${ }^{4}$ : clase textural a través del método de Bouyoucos y porcentaje de materia orgánica y carbono por medio del método de Walkley y Black. Para el análisis múltiple, las muestras fueron procesadas mediante el procedimiento especificado por Coil et al. (2003), el cual apunta a la extracción e interpretación múltiple de distinto tipos de microfósiles (polen, almidón, anillos de celulosa, microcarbones, silicofitolitos, cristales de calcio, esferulitas, crisofíceas, diatomeas y esporas) a través de la aplicación de un protocolo de bajo impacto químico.
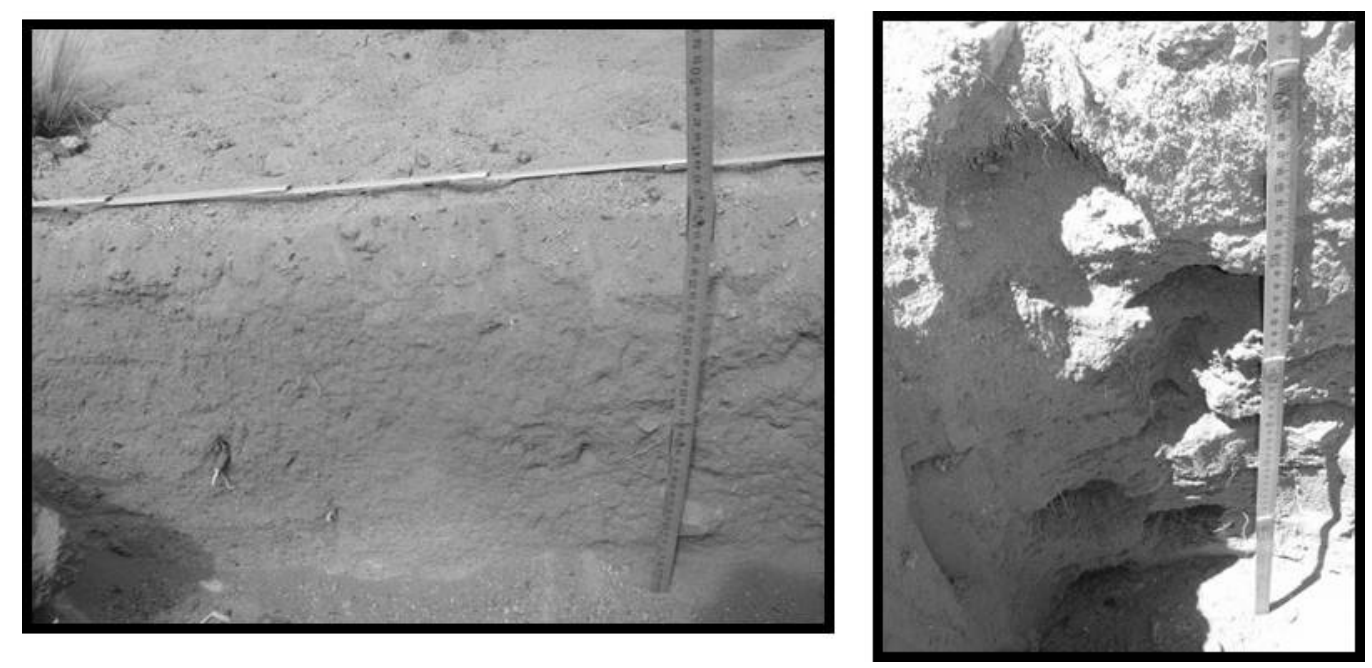

Figura 4. Imagen de perfiles de calicatas dentro de la estructura de cultivo 5 antes de ser muestreada (izquierda) y luego del muestreo (derecha) 
El análisis microscópico se realizó con microscopio petrográfico con luz transmitida y polarizador, a un aumento de 400x, observándose el $50 \%$ de los campos del cubre - objeto de cada muestra, registrándose las frecuencias relativas de los microfósiles detectados. Como ya mencionamos anteriormente tomamos como base interpretativa la propuesta de Korstanje y Cuenya $(2008,2010)$. Por otro lado, para las asignaciones taxonómicas de silicofitolitos y almidones de cultígenos, consultamos especialmente la colección de referencia de microfósiles de plantas económicas surandinas presentada por Korstanje y Babot (2007).

\section{Resultados de los análisis}

Por cuestiones de espacio disponible, no podremos presentar la totalidad de los resultados, razón por la cual nos enfocaremos en una única calicata (calicata 1 correspondiente a la estructura 5). En esta última, en todos los niveles se observó una preponderancia de fitolitos de la subfamilia Pooideae, seguidos de los correspondientes a Panicoideae y en último término Arundinoideae, salvo en el nivel superficial donde se encuentra mayor prevalecencia de los fitolitos de Arundinoideae sobre los de Panicoideae. La subfamilia Chloridoideae aparece escasamente registrada sólo en algunas de las capas. Las dicotiledóneas son representadas tanto por placas perforadas como así también por fitolitos esféricos faceteados afines a Cucurbitaceae. Éstos últimos están presentes en todos los niveles salvo en el superficial.

Asimismo, a lo largo de toda la secuencia, a excepción del nivel superficial, se detectaron gránulos de almidón afines a maíz y en los niveles correspondientes a los 15 a 30 cm y 45 a $60 \mathrm{~cm}$ se hallaron cruces diagnósticas de este cultígeno (según Piperno 1984, Piperno y Pearsall 1998, Pearsall et al. 2004). Por otro lado en el último nivel mencionado detectamos agrupaciones de almidón tipo "ojo de mosca" afines a los géneros Chenopodium y Amaranthus. Las esporas y los microcarbones corresponden a los microfósiles más numerosos en las muestras, luego de los silicofitolitos. Las diatomeas aparecen en mayor cantidad solamente en la muestra correspondiente al nivel superficial. Los anillos de celulosa se registraron en la mayoría de las muestras (salvo en la capa correspondiente a la superficie y entre los 45 a $60 \mathrm{~cm}$.).

En lo que refiere a los análisis pedológicos, observamos una fuerte homogeneidad en la clase textural (franco arenoso), la cual se mantiene en todo el perfil de la calicata. En lo que respecta a la textura gruesa del suelo (partículas de más de $2 \mathrm{~mm}$ ) caracterizada a campo, observamos que en todo el perfil muestreado, los elementos más gruesos eran poco frecuentes a diferencia de lo que ocurría en el perfil correspondiente a la calicata extra sitio, en donde fueron recurrentes las gravillas y clastos. Esto puede explicarse, posiblemente en razón de la actividad de despedre realizada en los campos agrícolas a la hora de preparar el terreno para la siembra.

Por otro lado, los análisis de materia orgánica arrojaron una clara homogeneidad en la clasificación ("Extremadamente Pobres", valor promedio: 0,45 \%), detectándose una disminución de materia orgánica en profundidad, aunque los valores se mantienen en los dos niveles más cercanos a la superficie. Si comparamos con los porcentajes del perfil extra sitio (que correspondería a un suelo no agriculturizado; valor promedio: $0,86 \%$ ), vemos una disminución del contenido de materia orgánica (los cuales se reducen a la mitad) en las muestras tomadas dentro de los canchones. 


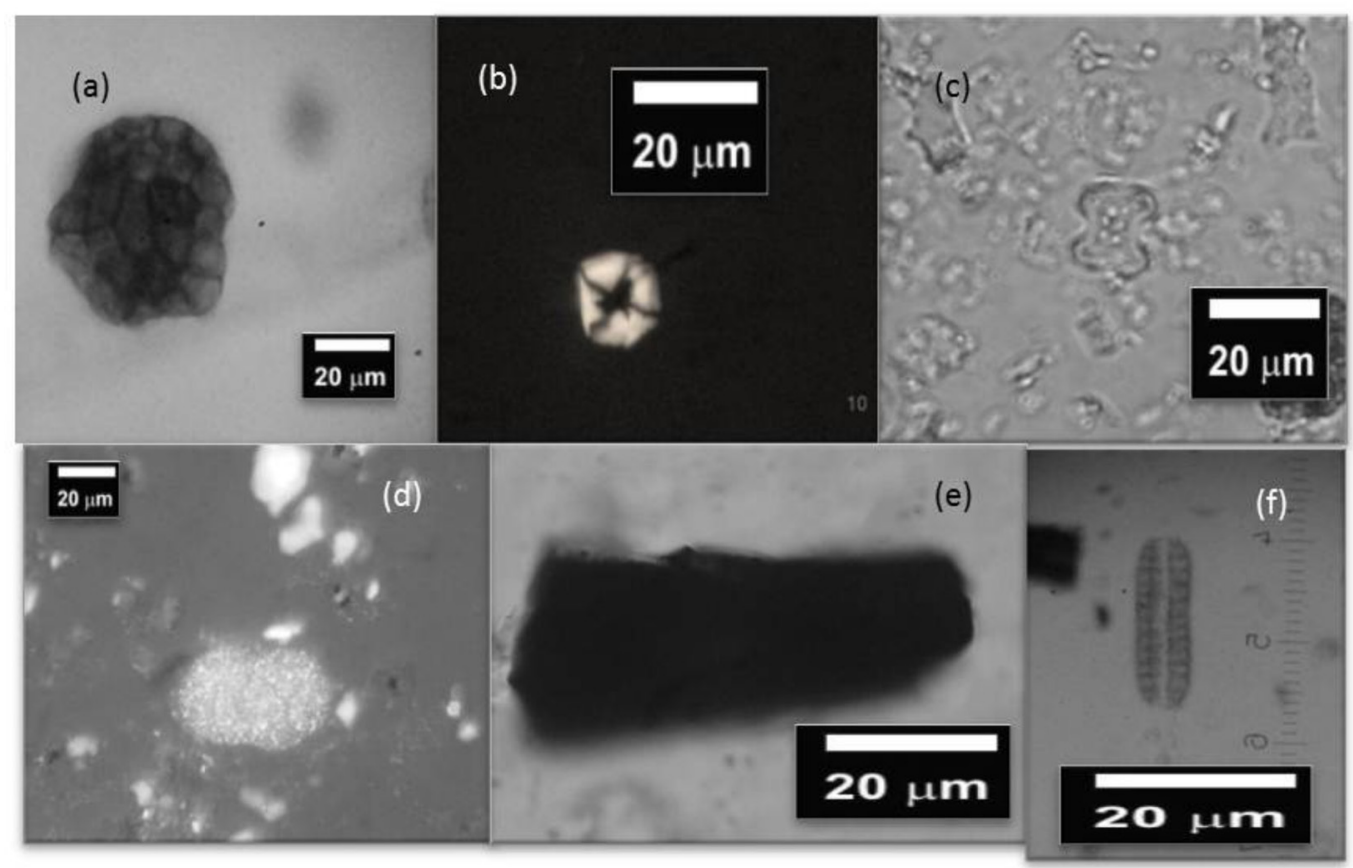

Figura 5. Microfósiles identificados en el sitio. (a) silicofitolito afín a Cucurbitaceae; (b) gránulo de almidón afín a Zea mays; (c) silicofitolito afín a Zea mays; (d) microalmidones afines a Chenopodium o Amaranthus; (e) microcarbón; (f) diatomea

A partir de lo expresado hasta aquí podemos extraer algunas conclusiones. En primer lugar observamos que en todas las muestras aparecen representados taxa silvestres que actualmente encontramos en la zona. Por otro lado, con respecto a los microfósiles afines a plantas cultivadas, no pudimos dejar de observar una posible asociación entre el cultivo de maíz y Cucurbitaceae (la cual se repite en otros niveles de las otras calicatas muestreadas).

En cuanto a las diatomeas contabilizadas, encontramos que éstas no solo aparecen con mayor frecuencia en los niveles superficiales, decreciendo en número en los más profundos, sino que el mayor conteo de las mismas se registró en las muestras extra sitio.

\section{Integrando resultados}

Hasta aquí hemos presentado por separado los resultados de diferentes análisis que llevamos a cabo en esta investigación, avanzado en el conocimiento de la arquitectura, conjuntos líticos, características pedológicas y microfósiles del Alto Juan Pablo, siendo éste el momento de integrar la información con el objetivo de caracterizar su taskscape.

\section{El taskscape del Alto Juan Pablo}

Volviendo sobre los resultados de los microfósiles e integrando la información pedológica, observamos en la calicata 1 (Estructura 5), indicadores del probable cultivo continuado de maíz y Cucurbitaceae. Sólo en una de las capas se registró la posible incorporación de Chenopodium o Amaranthus. Desde los análisis de materia orgánica vemos que los resul- 
tados del total de estas muestras indicaron una disminución de la misma en comparación con el perfil extra sitio. Esto, interpretado junto a los resultados del análisis de microfósiles (que, como veremos más adelante, indican laboreo del terreno, quema de rastrojos, cultivo continuado, ausencia de fertilización con guano), nos permite pensar que podría haber habido un proceso de agotamiento del suelo por cultivo intenso, lo cual aparece más acentuado en los niveles inferiores, es decir los mismos que presentan evidencias positivas de microfósiles afines a plantas cultivadas. Este proceso de agotamiento edáfico, conllevaría un retardamiento en la recuperación de la materia orgánica (especialmente frecuente en zonas de climas semi - árido a áridos), lo cual explicaría las diferencias entre los porcentajes de materia orgánica de las calicatas dentro de los canchones y la extra - sitio. Por otro lado, analizando los valores dentro del perfil de la calicata de la estructura 5, vemos una disminución de la materia orgánica en profundidad, encontrando los valores más bajos para todo el conjunto de muestras del sitio, en el nivel más profundo. Esto último lo interpretamos en razón de que no sólo se trata de un suelo probablemente agotado en el pasado, sino también el más viejo ya que coincidentemente es el más profundo de todas las calicatas realizadas. En los dos niveles más superficiales encontramos valores similares de materia orgánica. Esto no se corresponde con un perfil tipo, no antropizado, ya que en este caso lo esperable es la disminución de contenidos de materia orgánica, lo cual no sucede. Hasta ahora no tenemos explicación para esta "anomalía" ${ }^{5}$. Hubiese sido posible explicar este comportamiento atípico de la materia orgánica a través de la incorporación de guano como fertilizante, pero dado que no hallamos esferulitas en ninguna de las muestras, la idea de fertilización con guano parece no poder ser sustentada.

Con respecto a las diatomeas, sólo encontramos un conteo superior en el nivel superficial a los registrados en esta cata, mientras que en los restantes niveles las mismas aparecían en igual o menor cantidad. Este dato lo consideramos de gran importancia para volver sobre el tema del riego. Si consideramos a los valores obtenidos en las muestras extra sitio como indicadores de condiciones no cultivadas (y por ende no bajo riego), los conteos superiores de diatomeas podrían ser tomadas como posible evidencia de riego (Korstanje y Cuenya $2008,2010)$. Esto no sucedió en ninguna de las muestras con asociación a microfósiles afines a algún cultígeno. La única muestra que supera claramente el conteo de las silicoalgas del extra sitio, corresponde a la de superficie donde no se han hallado, desde los microrrestos, evidencias de taxones cultivados. Esto nos estaría indicando la probable ausencia de riego, idea que se refuerza ante la no identificación de células silíceas articuladas (Miller Rosen 1992, Miller Rosen y Weiner 1994, Madella 2007, Mithen et al. 2008), como también ante la no detección de estructuras destinadas a la irrigación (ver supra). Frente a esto, es interesante volver sobre la recurrente asociación observada de microvestigios de maíz y Cucurbitaceae. Así, según datos ofrecidos por los propios campesinos, dicha combinación es frecuente en situaciones de cultivo a secano, ya que, por un lado el tallo del maíz sirve de guía o tutor para el zapallo, al mismo tiempo que éste último genera una especie de capa protectora del suelo en la cual no sólo se reduce el impacto de la gota de lluvia en la superficie sino fundamentalmente mantiene la humedad por más tiempo y reduce la evapotranspiración y por ende la pérdida de agua, ayudando así al maíz cuyos requerimiento hídricos no son tan bajos como los de algunas Cucurbitáceas.

Por otro lado, los bajos valores de materia orgánica, sumado a la abundancia de fitolitos y de microcarbones presentes en numerosas muestras con evidencias positivas de microvestigios afines a taxa cultivados, puede estar indicando la quema de rastrojos (Korstanje y Cuenya 2008, 2010). Si bien somos conscientes de que los microcarbones pueden haber 
sido aportados eólicamente, la presencia de este tipo de partículas de gran tamaño y bordes angulosos, refuerzan la hipótesis de incendios in situ, ya que aquellos que son trasladados por el viento presentan menores dimensiones y bordes redondeados.

Otra observación interesante corresponde al gran número de microfósiles fragmentados que fueron observados en las muestras, lo cual puede ser concordante con la práctica de labranza de los campos, la cual afecta mecánicamente a los microvestigios. Esto puede reforzarse por los valores bajos de materia orgánica, ya que una fuerte actividad de labranza afecta desfavorablemente los contenidos de esta materia en el suelo (Shaxson y Barber 2005).

Hasta aquí hemos presentado un esbozo de las prácticas productivas, las cuales habrían incluido la labranza de los campos, la quema de rastrojos y la combinación de cultivos en el marco de una agricultura a secano y en ausencia de abonado con guano. Si a este panorama incluimos la información obtenida a partir de los análisis del conjunto lítico, observamos que estas actividades vinculadas a la producción agrícola pudieron combinarse con otras como ser la regularización o reformatización de artefactos confeccionados fuera del sitio. Así, podemos proponer que parte del tiempo que trascurría en las parcelas se consumía en tareas de mantenimiento de instrumentos de labranza (lo cual podría estar representado por el gran número de lascas de gran tamaño recolectadas).

Un punto sobre el cual nos detuvimos con anterioridad, refiere a la posible secuencia de crecimiento de las estructuras (ver supra), a partir de la cual propusimos que podría tratarse de un diseño de expansión del sector agrícola orientado al aprovechamiento de espacios dejados por las estructuras anteriores, resultando de estas decisiones constructivas, un modelo de crecimiento irregular y episódico. Lamentablemente nos es imposible establecer en qué momento fue construido cada canchón, sin embargo, podríamos aventurarnos a pensar que esta modalidad particular de crecimiento, no es discordante con la necesidad de dejar bajo barbecho las parcelas cuyo suelo se habría agotado. Dicha necesidad puede haber sido resuelta incorporando nuevos canchones que son cultivados mientras los anteriores quedan en descanso. Una vez recuperadas las condiciones de éstos, pueden reactivarse, posiblemente permitiendo el barbecho de los construidos posteriormente. De esta manera el espacio agrícola se estructuraría según lo que conocemos como barbecho sectorial. Sin embargo no queremos dejar de resaltar el carácter especulativo de esta interpretación, basada únicamente en observaciones actuales (ya que en Nacimientos de San Antonio encontramos un manejo de las parcelas similar al descripto).

Finalmente, en algún momento posterior a la construcción del Alto Juan Pablo, se registraron cambios en la dinámica geomorfológica, siendo invadido por dunas provenientes del área puneña. "Esta invasión de dunas solo puede ser explicada por una combinación de factores (climáticos, eventos volcánicos con producción de abundante material piroclástico y/o antrópicos) que deberán ser analizados en contextos regionales" (Kulemeyer et al. 2012: 24). En cualquier caso, los efectos sobre el entorno del sector norte del valle, habrían instaurado nuevos desafío para los campesinos que allí habitaban.

Con todo lo anterior no pretendemos pensar que el taskscape del Alto Juan Pablo se reduce únicamente a las actividades que pudimos interpretar. Somos conscientes que aquí presentamos sólo una versión parcial y fragmentada de una imagen que seguramente fue mucho más compleja y rica. 


\section{Incorporando El Alto Juan Pablo al paisaje agrario del sector septentrional}

Hasta aquí, hemos buscado integrar todos los resultados de los análisis realizados con el objetivo de construirnos una imagen del Alto Juan Pablo, específicamente de las prácticas objetivadas en su materialidad, a través de las cuales pensamos su taskscape. En esta oportunidad, nos disponemos a incorporar el sitio al paisaje del sector septentrional del valle o mejor dicho al conocimiento que de él tenemos gracias a las investigaciones precedentes allí efectuadas (Korstanje 2002, 2005, 2007, 2009). Para ello, tomaremos específicamente los estudios realizados por la citada autora en torno a dos sitios agrarios del sector septentrional (la Mesada - Morro Relincho y El Alto El Bolsón), los cuales han sido abordados con la misma metodología de análisis múltiples aquí aplicada, lo cual permite comparar resultados. Esto nos ha permitido discutir tres cuestiones de especial relevancia para la arqueología de la región: 1) la desencialización de la agricultura formativa (algo ya abordado en otros trabajos como ser Korstanje et al 2012), 2) la relación entre los campesinos y la estructura ambiental y 3) la organización socio-política del paisaje agrario de la región.

Con respecto al primer ítem, al cruzar las interpretaciones vinculadas a las prácticas agrícolas del Alto Juan Pablo con aquellas propuestas por Korstanje para los sitios mencionados, observamos que para el caso de La Mesada/ Morro Relincho (LMMR en adelante), Korstanje ha identificado evidencias de cultivo de maíz, quínoa y probablemente ulluco en las parcelas y de procesamiento de maíz, Cucurbitaceae, y tubérculos en contexto residencial. A pesar que el maíz no es una espacie que sea esperable encontrar siendo cultivada a secano, la autora no identificó evidencias de riego (ni arquitectónicas ni a través de diatomeas), tampoco detecta indicios de que los campos hayan sido abonados, ni intensamente despedrados. Asimismo, descarta aquí la idea de una agricultura y barbecho con uso ganadero. El panorama para El Alto El Bolsón (EAEB en adelante) presenta algunas diferencias. En contexto residencial Korstanje identifica microfósiles de zapallo, maíz y quínoa o amaranto, mientras que en las parcelas detecta microvestigios de tubérculos en niveles superficiales y de maíz en los inferiores, sugiriendo una posible rotación de cultivos. Por otro lado, los niveles superiores (asociados a almidones de tubérculos) parecieron haber sido fertilizados por abonado. Si bien en EAEB existen estructuras de riego, las mismas corresponderían a sectores post - formativos.

El Alto Juan Pablo, en relación a los dos sitios a los que nos hemos referido, presenta algunas diferencias interesantes que nos hacen pensar que la producción agrícola pareció incluir diversas prácticas que mediaron entre los campesinos y los campos del sector septentrional del valle. Así, por ejemplo el Alto Juan Pablo presentó evidencias que sugieren una asociación de cultivos diferente a las observadas en los otros dos sitios (ver supra). Por otro lado, la rotación de cultivos observada por Korstanje en EAEB, no fue identificada en LMMR ni en Alto Juan Pablo. En lo que refiere a la práctica de fertilización con guano, tampoco aparece como una constante entre los sitios formativos de la zona, ya que solo fue reconocida en uno de los sitios analizados (EAEB), no así en los demás emplazamientos agrícolas del valle. En lo que refiere a los aspectos constructivos, observamos que la construcción arquitectónica de las parcelas, también se resolvió de manera diferente. Así, mientras en Alto Juan Pablo tenemos un diseño de adosamiento de canchones sin solución de continuidad, el cual también aparece en algunos sectores de EAEB, sin embargo para LMMR, Korstanje registra decisiones constructivas diferentes (parcelas individuales y otras adosadas en no más de dos estructuras). Con respecto al riego, los tres sitios formativos hasta aquí mencionados carecen de evidencias de irrigación pero todavía no podemos sugerir que la agricultura a 
secano haya sido una constante durante el Formativo ya que existe un registro mencionado por Korstanje (2005) de un sitio agrícola de este momento posiblemente irrigado, nos estamos refiriendo a Los Llampa. Todo esto nos permite proponer (por lo pronto como hipótesis a ser chequeada a través de futuros trabajos) que el paisaje del sector septentrional del valle de El Bolsón queda configurado desde la variabilidad en las estrategias productivas durante el Formativo. Así, nos parece que no existió durante este momento un único modo estandarizado para la agricultura en la zona, sino más bien diferentes prácticas productivas eran convocadas por los campesinos, las cuales incluyeron distintas decisiones en relación a los taxones cultivados, rotación y asociación de cultivos, abono con guano, etc. Entonces, estamos pensando en un Formativo del sector septentrional en el que coexistieron conocimientos de diferentes técnicas agrícola los cuales se usaron alternativamente.

El segundo ítem mencionado en párrafos precedentes, refiere a la relación entre los campesinos y la estructura ambiental. En este punto cobra especial relevancia el hecho de que en el sector septentrional del valle se reconocieron, a parte de los sitios agrícolas adscriptos al Formativo que ya mencionamos, otros de cronologías más tardías (es el caso de Yerba Buena y Vaca Vizcana 3, ambos ubicados en los Desarrollos Regionales). La razón por la cual consideramos relevante incorporar esta información es que, si tenemos en cuenta las reconstrucciones paleoambientales propuestas para el valle (ver supra), observamos a través de los estudios de Ortíz (2001) y Madozzo (2009) que de hecho han ocurrido cambios en las condiciones ambientales pretéritas, las cuales no debieron pasar desapercibidas para los campesinos prehispánicos del lugar. Sin embargo, la presencia de sitios agrícolas durante todo el período agroalfafrero en la zona, ha logrado evidenciar una continuidad en la producción agrícola. De esto, podemos aventurarnos a sugerir que las modificaciones en la estructura ambiental, si bien habrían influido, no conllevaron el fin de la agricultura en la región. En este punto sería interesante contar con información de detalle de varios sitios que nos permitan comprender los procesos de negociación entre los campesinos y estas modificaciones en el entorno ambiental, sin embargo no contamos aún con esta posibilidad. Lo que sí sabemos es que, esa negociación debió existir para poder continuar con la producción agrícola, siendo las prácticas productivas las mediadoras entre los campesinos y la estructura ambiental.

Por otro lado, esa misma capacidad de negociación la vemos en relación a las unidades geomorfológicas seleccionadas para la construcción de las parcelas agrícolas. Así, encontramos emplazamientos productivos en terrazas fluviales asociadas a cursos de agua permanente (Los Llampa), en laderas de montañas con fuertes pendientes sin acceso a agua para riego (LMMR) y en mesadas de elevaciones (EAEB y Alto Juan Pablo). En esto vemos una adecuación de la agricultura a diferentes condiciones geomorfológicas posiblemente resuelta a través de las técnicas constructivas de los espacios productivos, lo cual podría explicar las diferentes configuraciones arquitectónicas que éstos adoptaron durante el Formativo. Una vez más vemos a las prácticas (en este caso de construcción de espacios agrícolas) mediando en el diálogo entre los campesinos y el entorno físico, resultando de este diálogo el paisaje agrario formativo.

A partir de lo anteriormente expuesto, estamos pensando en campesinos construyendo el paisaje productivo prehispánico no como sujetos desprovistos de agencialidad, respondiendo ante los condicionamientos de la estructura ambiental, sino como sujetos provistos de una gran versatilidad técnica que les permite plantear diferentes estrategias frente a las restricciones y habilitaciones de su medio. 
En relación a la tercera cuestión que mencionamos, nos preguntamos acerca del grado de autonomía familiar de las sociedades formativas del sector septentrional del valle. Con respecto a este tema, Korstanje $(2005 ; 2007)$ presenta una imagen del territorio formativo del valle en el que no se observa desigualdad social, sino más bien campesinos dedicados con más en énfasis a las tareas productivas primarias, sin evidencia de una supra - organización por parte de un poder centralizado. Esta imagen parece persistir durante todo el Formativo, sin reconocerse indicadores ni de defensa ni de sometimientos (ni en el arte rupestre como así tampoco en la construcción y uso de los espacios) (Korstanje 2005, 2007). Si tenemos en cuenta lo expuesto en párrafos precedentes respecto a la variabilidad entre los sitios formativos, en lo que a las prácticas agrarias refiere y técnicas constructivas de los espacios a ser cultivados, nos enfrentamos a un paisaje muy heterogéneo que no nos hace pensar en una elite gestionando la producción agrícola, ya que si la decisión productiva se hallaría centralizada esperaríamos entonces una mayor estandarización y homogeneidad en esas decisiones. Más bien nos parece que cada familia extensa está administrando, controlando, construyendo, etc. sus propios espacios productivos, recayendo en ellas las decisiones y ejecución de la producción. Esta idea puede reforzarse, si tomamos en cuenta la gran descentralización de los espacios productivos del Primer Milenio, ya observada por Korstanje (2005), ya que bajo un poder centralizado supra familiarmente, sería para nosotros, más esperable que dicha centralización se refleje en un paisaje agrícola concentrado y no disperso como parece ser el caso del sector septentrional de El Bolsón.

Finalmente, si pensamos en la escala en que se habría gestionado el emplazamiento Alto Juan Pablo, podemos pensar que su construcción no habría requerido la movilización y coordinación de una gran fuerza de trabajo que supere las posibilidades de una unidad doméstica. Esta idea parece afianzarse si tenemos en cuenta que el complejo de estructuras no necesariamente debió ser construido en un mismo momento, como puede sugerirse a partir de la secuencia constructiva del sitio que hemos propuesto.

\section{Palabras finales}

Hasta aquí, estamos pensando en el Alto Juan Pablo como un emplazamiento que comienza a ser construido en algún momento del Formativo, configurándose como un espacio agrícola constituido por algunas estructuras adosadas entre sí. Éstas habrían sido construidas con rocas de las inmediaciones de variados tamaños, siendo apiladas para levantar sus muros, generando así una protección contra la erosión del suelo. La preparación de los suelos pudo incluir el despedrado (evidenciado tal vez por las observaciones realizadas de la textura gruesa del suelo) y la labranza previa a la siembra (posiblemente indicado por la alta frecuencia de microrrestos fragmentados). Antes, durante o después de esta labranza, se pudieron reactivar in situ los instrumentos líticos utilizados en esta actividad. El calendario agrícola, adaptado a las particularidades de cada año, habría guiado la siembra, mantenimiento y cosecha de los cultivos. Luego de esta última, los rastrojos habrían sido quemados, quedando el campo a disposición para un nuevo ciclo. Sucesivos cultivos podrían haber llevado al agotamiento de los suelos, frente a lo cual se podrían haber incorporado nuevas parcelas. De esta manera el Alto Juan Pablo iría adoptando la configuración que hoy presenta, mediando desde su abandono como emplazamiento de producción agrícola hasta nuestros días un sinnúmero de contextos desde los cuales el sitio habría sido resignificado. 
Agradecimientos: A mis directores Dra. Korstanje y Dr. Quesada (por sus invaluables aportes y comentarios durante la realización de mi tesis, parte de la cual presentamos en este artículo), a quienes colaboraron durante los trabajos de campo, al CIUNT y CONICET (por el incentivo que implican las becas) y al Instituto de Arqueología y Museo (por abrirme siempre las puertas). Finalmente a los evaluadores, cuyas sugerencias y aportes mejoraron notablemente la versión original de este trabajo. Los errores son de mi exclusiva responsabilidad.

\section{Notas}

1. A esto debemos sumar que la visibilidad superficial se vio afectada por un médano que cubre parte del sector aledaño al sitio.

2 Para el caso particular del basalto, Somonte (2004), lo incluye como recurso local, pudiéndose encontrar por ejemplo formando parte de afloramientos de depósitos Cuartarios (sic), en forma de rodados grandes, medianos y pequeños. Estos depósitos, se presentan como capas cubriendo sectores de las elevaciones montañosas, y remanentes de terrazas (Babot y Larrahona 2001). En las cercanías del sitio aquí estudiado, Somonte menciona su presencia en el sitio La Mesada el cual se encuentra en la misma localidad que El Alto Juan Pablo. Sin embargo cabe aclarar que para determinar la exacta proveniencia de las materias primas de los artefactos estudiados se requerirían análisis de detalle que no han sido realizados en esta oportunidad.

3. La elección de la localización de la calicata extra sitio, se realiza buscando mantener lo más estandarizadas posible las variables ambientales (por ejemplo relieve, condiciones microambientales, etc.), las cuales podrían introducir un sesgo en la comparación con las muestras provenientes del interior de las estructuras, que conllevarían errores interpretativos. 4. Estos análisis fueron llevados a cabo por la Geol. Patricia Cuenya.

5. Si bien se podría pensar en un uso agrícola reciente de estas estructuras para explicar estas "anomalías", las consultas efectuadas a los pobladores de la localidad de Nacimientos de San Antonio, no nos permiten sostener esto.

\section{Bibliografía Citada}

Albeck, M. E.

1993 Contribución al estudio de los sistemas agrícolas prehispánicos de Casabindo (Puna de Jujuy). Tesis doctoral no publicada, Facultad de Ciencias Naturales y Museo, Universidad Nacional de la Plata, La Plata.

Álvarez, S.

2001 Uso del Espacio para la producción agrícola y vida rural de grupos de filiación aguada en la quebrada de El Tala. Tesis de Licenciatura no publicada, Escuela de Arqueología, Universidad Nacional de Catamarca, Catamarca.

Aschero, C.

1975 Ensayo para una clasificación morfológica de artefactos líticos aplicada a estudios tipológicos comparativos. Buenos Aires. Ms

1983 Ensayo para una clasificación morfológica de artefactos líticos aplicada a estudios tipológicos comparativos. Apéndices A-C. Revisión 1983. Cátedra de Ergología y Tecnología. Facultad de Filosofía y Letras. Universidad de Buenos Aires. (Inédito) 
Aschero, C. y S. Hocsman

2004 Revisando cuestiones tipológicas en torno a la clasificación de artefactos bifaciales. Temas de arqueología. Análisis lítico (comp. por Ramos, Acosta y Loponte), pp. 7-26. Universidad Nacional de Luján

Aschero, C. y M.A. Korstanje.

1996 Sobre figuraciones humanas, producción y símbolos. Aspectos del arte rupestre del Noroeste Argentino. Volumen XXV Aniversario del Museo Arqueológico Dr. Eduardo Casanova, pp: 13-31. Instituto interdisciplinario Tilcara. (UBA). Jujuy

Babot, M. del P. y P. Larrahona

2001 Artefactos de molienda y materias primas en los valles del Noroeste. Actas del XIV Congreso Nacional de Arqueología Argentina. Facultad de Humanidades y Artes, Escuela de Antropología, Universidad Nacional de Rosario, Rosario.

Burkart, R.; Bárbaro, N.O.; Sánchez, R.O. y D.A. Gómez

1999 Eco-regiones de la Argentina. Administración de Parques Nacionales. Programa Desarrollo Institucional Ambiental. Buenos Aires

Caria, M. A, Oliszewski, N., Pantorrilla, M. y J. Gómez Augier.

2007 Relevamiento y clasificación del sistema agrícola prehispánico en la quebrada del río los Corrales (El Infiernillo, Tafí del Valle, Tucumán). Pacarina Número especial del XVI Congreso Nacional de Arqueología Argentina, I: 49-54, Jujuy.

Coil, J., Korstanje, M.A., Archer, S. y C.A. Hastorf

2003 Laboratory goals and considerations for multiple microfosil extration in archaeology. Journal of Archaeological Science - 30: 991-1008. Academic Press.

Descola, P.

2001 Construyendo naturalezas. ecología simbólica y práctica social. Naturaleza y Sociedad. Perspectivas Antropológicas, pp. 101-124 (ed. por Descola y Palsson). Siglo XXI

Díaz, R. A.

2009 Historias de tierra y agua: Introducción a los espacios agrícolas prehispánicos de Laguna Blanca. El caso de estudio de la Aldea Arqueológica Piedra Negra (Dpto. Belén, Provincia de Catamarca). Tesis de Licenciatura no publicada, Escuela de Arqueología, Universidad Nacional de Catamarca.

Escola, P., Babot, M del P. y S. Hocsman

2012 Contribuyendo a re - pensar a los "pastores" puneños: los cuchillos - raederas de módulo grandísimo como caso. En Arqueología del Período Formativo en Argentina. Un Encuentro para integrar áreas y sub-disciplinas, revisar significados y potenciar el impacto de las investigaciones en curso. Tafí del Valle, Tucumán

Figueroa, G.

2008 Los sistemas agrícolas del Valle de Ambato, Catamarca, siglos VI a XI d.C. Intersecciones en Antropología 9: 313-317. 
Franco Salvi, V.

2012 Estructuración social y producción agrícola prehispánica durante el Primer Milenio D.C. en el Valle de Tafí (Tucumán, Argentina). Tesis Doctoral no publicada, Facultad de Filosofía y Humanidades, Universidad Nacional de Córdoba.

Haber, A. F.

1999 Una Arqueología de los Oasis Puneños. Domesticidad, interacción e identidad en Antofalla, Primer y Segundo Milenio d.C. Tesis Doctoral no publicada en Filosofía y Letras, Facultad de Filosofía y Letras de la Universidad Nacional de Buenos Aires

Ingold, $\mathrm{T}$.

1993 The Temporality of the landscapes. World Archaeology: conceptions of time and ancient society Vol 25: 189-208

2000 The perception of the enviroment. Essays on livelihood, dwelling and skill. Routledge London

Korstanje, M.A.

2002 Microfósiles y agricultura prehispánica: primeros resultados de un análisis múltiple en el N.O.A. En: Análisis fitolíticos de vegetación, suelos, sedimentos y sitios arqueológicos: estado actual de su conocimiento en América del Sur (ed. por Zucol, A.F.; Osterrieth, M.; Brea, M. y $\mathrm{N}$. Borrelli). En prensa.

2005 La organización del trabajo en torno a la producción de alimentos en sociedades formativas (Provincia de Catamarca, República Argentina). Tesis Doctoral no publicada, Instituto de Arqueología y Museo, Facultad de Ciencias Naturales e I.M.L. U.N.T. Tucumán, Argentina

2007 A Microfossil characterization from South Andean economic plants. Plants, People and places: recent studies in phytolithic analysis, proceeding of The 4th International Meeting on Phytolith Research, (ed. por M. Madella y D. Zurro), pp:........ . Cambridge, Oxbow Books. 2009 Microfósiles y agricultura prehispánica: primeros resultados de un análisis múltiple en el N.O.A. Fitolitos: estado actual de su conocimiento en América del Sur (ed. por Zucol; Osterrieth y Brea), pp. 249-263. Universidad Nacional de Mar del Plata.

2010 IAM en los Valles Altos del Oeste Catamarqueño. Rastros en el camino... Trayectos $e$ identidades de una institución. Homenaje a los 80 años del IAM - UNT. (Comp. por Arenas, P., C. Aschero y C. Taboada), pp: 295-302. EDUNT. Universidad Nacional de Tucumán.

Korstanje, M. A y M. P. Babot.

2007 A Microfossil Characterization from South Andean Economic Plants. En: Plants, People and Places: Recent Studies in Phytolithic Analysis, Proceeding of The 4th International Meeting on Phytolith Research, editado por M. Madella y D. Zurro. Cambridge, Oxbow Books.

Korstanje M. A. y P. Cuenya.

2008 Arqueología de la agricultura: suelos y microfósiles en campos de cultivo del valle del Bolsón, Catamarca, Argentina. En: Matices interdisciplinarios en estudios fitolíticos y de otros microfósiles. BAR International Series 1870, (ed. por A. Korstanje y P. Babot), pp. 133-147.

2010 Ancient agriculture and domestic activities in north western Argentina: a contextual approach studying silicaphytoliths and other microfossils in soils. Journal of Environmental Archaeology, Vol 15, № 1: 43-63. The Charlesworth Group, Wakefield. 
Korstanje, A.; Cuenya, P. y M. Maloberti

2012 El análisis múltiple de microfósiles como herramienta para estudiar paisajes agrícolas y prácticas campesinas: una síntesis metodológica. En: Avances y desafíos metodológicos en arqueobotánica: miradas consensuadas y diálogos compartidos desde Sudamérica (ed. por Belmar, C. y V. Lema). En prensa

Korstanje, M.A.; Quesada, M.N.; Franco Salvi, V.L y M. Maloberti.

2012 ¿Cómo son los Paisajes Agrarios del Formativo? Trabajo pre - circulado en Arqueología del Período Formativo en Argentina. Un Encuentro para integrar áreas y sub-disciplinas, revisar significados y potenciar el impacto de las investigaciones en curso. Tafí del Valle, Tucumán

Kulemeyer, J.; Lupo, L.; Madozzo, C.; Cruz, A.; Cuenya, P.; Maloberti, M.; Cortés, G. y A. Korstanje.

2012 Desarrollo del Paisaje Holoceno en la Cuenca de El Bolsón: gente y ambiente en procesos de cambio y estabilidad, Trabajo presentado en el $54^{\circ}$ Congreso Internacional de Americanistas. Viena, Austria.

Madella, M.

2007 The Silica Skeletons from the Anthropic Deposits. En: The Early Neolithic on the Great Hungarian Plain Investigations of the Körös culture site of Ecsegfalva 23, County Békés. (ed. por A. Whittle). Academy of Sciences. Budapest, pp. 447-460.

Madozzo Jaen, M.C.

2009 Micromamíferos del Holoceno Tardío: Taxonomía, tafonomía y reconstrucción paleoambiental. Tesina de grado no publicada, Facultad de Ciencias Naturales e Instituto Miguel Lillo, Universidad Nacional de Tucumán

Miller Rosen, A.

1992 Phytoliths as indicators of ancient irrigation farming. En: Préhistoire de l'agriculture: nouvelles approaches experimetales et etnographiques. (ed. por Anderson), Monographie du CRA No 6: 281-287. Editions du CNRS. París.

Miller Rosen, A. y Weiner, S.

1994 Ancient Irrigation: a new method using opaline phytoliths from Emmer Wheat. Journal of Archaeological Science 21:125 - 132.

Mithen, S.; Jenkins, E. L.; Jamjoum, K.; Nuimat, S.; Nortcliff, S. y B.L. Finlayson 2008 Experimental crop growing in Jordan to develop a methodology for the identification of ancient crop irrigation. World Archaeology, 40:7-25.

Morgan, R.P.C.

1986 Soil erosion and conservation. Longman. Nueva York.

Ortiz, P.E.,

2001 Roedores del Pleistoceno Superior del Valle de Tafí (Provincia de Tucumán), Implicancias paleoambientales y paleobiogeográficas. Tesis doctoral no publicada, Facultad de Ciencias Naturales e Instituto Miguel Lillo, Universidad Nacional de Tucumán 
Pearsall, D.

2000 Paleoethnobotany. A handbook of procedures. Academic Press

Pearsall, D.; Chandler - Ezell, K. y Chandler - Ezell, A.

2004 Maize can still be identified using phytoliths: response to Rovner. Journal of Archaeological Science 31:1029-1038. Academic Press

Piperno, D.R.

1984 A comparison and differentiation of phytoliths from maize and wild grasses: use of morphological criteria. American Antiquity 49(2): 361 - 383

1988 Phytolith Analysis. An Archaeological and Geological Perspective. Academic Press.

Piperno, D.R. y D. Pearsall

1998 The silica bodies of tropical american grasses: morphology, taxonomy, and implications for grass systematics and fossil phytolith identification. Smithsonian Contributions to Botany 80: 40

Quesada, M. N.

2007 Paisajes Agrarios del Área de Antofalla. Procesos de trabajo y escalas sociales de la producción agrícola. (Primer y Segundo Milenios D.C.) Tesis Doctoral no publicada, Fac. De Ciencias Naturales Y Museo, Universidad Nacional De La Plata

Quesada, M.N. y M. Maloberti

2012 La expansión de los espacios agrícolas. Continuidades en la construcción del paisaje agrario en el sector septentrional del Valle de El Bolsón (siglos I a XV). Racionalidades campesinas en los Andes: pasado, presente... (ed. por Cruz, P., Joffre, R. y Th. Winkel). Institut de Recherche pour le Développement. Montpellier. En prensa.

Shanks, M. y C. Tilley

1992 Social Archaeology: the Object of Study. Re-Constructing Archaeology. Theory and Practice. Routledge, London.

Shaxson, F. y R. Barber.

2005 Optimización de la humedad del suelo para la producción vegetal. el significado de la porosidad del suelo. Boletín de Suelos de la FAO. Roma.

Somonte, $\mathrm{C}$.

2004 Informe preliminar del análisis tecno-morfológico y tipológico de los conjuntos liticos de la microrregion del Valle del Bolson, Catamarca. Informe Agencia Nacional de Promoción Científica. Tucumán. 